\title{
Volume-Expansion Factor of ABA Block Copolymer
}

\author{
Homare Endo and Misazo Yamamoto \\ Faculty of Science, Tokyo Metropolitan University, \\ Setagaya, Tokyo, Japan.
}

(Received November 27, 1973)

\begin{abstract}
Some recent experiments show that the intrinsic viscosities of ABAtype triblock copolymers are sometimes smaller than those of the precursors $\mathrm{B}$ in the $\theta$ solvent for side blocks A.

To explain this fact, a triblock copolymer is represented by three spherical segmental distributions connected with each other by four freely jointed sticks.

The partition function is evaluated by the Bragg-Williams approximation with the lattice model. Expressions for the free energy and the volume-expansion factor of ABA-type triblock copolymers are obtained and conformational anomalies which have an effect upon the intrinsic viscosities are discussed by comparing the interaction energy and the unperturbed radius of gyration in the ring conformation with those for the averaged case.

KEY WORDS ABA Triblock Copolymer / Radius of Gyration / Lattice Model / Bragg-Williams Approximation / Volume-Expansion Factor / Intrinsic Viscosity /
\end{abstract}

During the past ten years or more many interesting experiments have been reported ${ }^{1-10}$ about the behavior of block copolymers in dilute solution. Much of the effort had been devoted since then to the synthesis and the isolation of block copolymers from the reaction product rather than to the solution properties, for it had been difficult to prepare polymers with narrow distributions in molecular weight and composition.

Experiments about the dilute solution behavior of AB-type (poly(methyl methacrylate)polystyrene) diblock copolymers were reported to suggest an intramolecular phase separation in a certain composition range under proper conditions and even the formation of intermolecular aggregates. ${ }^{9,10}$

Measurements of the intrinsic viscosity $[\eta]$ of ABA-type (poly(methyl methacrylate)-polystyrene-poly(methyl methacrylate)) triblock copolymers were made ${ }^{9}$ which implied also an intrachain phase separation: the ordinary random coil structure of the side-block copolymers collapses in their $\theta$ solvents below the $\theta$ temperature, resulting in intrachain association, with the central block being closed to a ring conformation due to aggregated strands of the two side blocks. For high-molecular-weight samples, the $[\eta]$ values of ABA-type triblock copolymers are reported to be smaller in the $\theta$ solvent for side blocks $A$ than those of the precursors $B$, which possess only about half the molecular weight or chain length of the whole block copolymer chain.

To explain this fact expressions for the free energy and the volume-expansion factor of ABA-type triblock copolymers are obtained and some conformational anomalies are discussed.

Flory's equation ${ }^{11}$

$$
\alpha^{5}-\alpha^{3}=C(1-\theta / T) M^{1 / 2}
$$

is used here, where $M$ is the molecular weight, $T$ is the temperature, $\theta$ is the function of the solvent quality, and $\alpha$ is the so-called volumeexpansion factor.

One of the methods for determining the dimensions of macromolecules in dilute solutions involves the observation of the intrinsic viscosity number $[\eta]$

$$
[\eta]=\Phi\left\langle s^{2}\right\rangle^{3 / 2} / M=[\eta]_{\theta} \alpha^{3}
$$

where $\Phi$ is a constant thought to be the same for all systems and $[\eta]_{\theta}$ is the intrinsic viscosity under $\theta$ conditions. [ $\eta]$ increases monotonously with the molecular weight $M$. 


\section{THE ESTIMATION OF THE MODEL}

In order to make use of the lattice theory, a triblock is represented by three spherical segmental distributions connected to each other by four sticks (Figure 1). One end of each stick is at the center of a sphere and the other is freely jointed with the next stick to connect two neighboring polymer blocks. This model has been suggested by Chujo and Yamamoto. ${ }^{12}$ The radius of each sphere is presumed to be equal to the radius of gyration of the corresponding polymer block, that is, $\left(n b^{2} / 6\right)^{1 / 2}$ for side blocks and $\left(m b^{\prime 2} / 6\right)^{1 / 2}$ for the central block, where $n, b, m$, and $b^{\prime}$ are the number of segments and the bond length of polymer $A$ and of polymer B, respectively. To avoid unnecessary complication, we assume here that the numbers of segments in the two side blocks are the same. The length of a stick is taken to be equal to the root-mean-square distance between the center of mass and an end of each polymer block.

$$
A=\left(n b^{2} / 3\right)^{1 / 2}, \quad B=\left(m b^{\prime 2} / 3\right)^{1 / 2}
$$

The calculation of the mean-square radius of gyration of a diblock copolymer for a similar model shows the same value as the exact one.

The mean-square radius of gyration of an ABA triblock copolymer for this model can be calculated to be

$$
\begin{aligned}
\left\langle s^{2}\right\rangle= & \left\{2 n^{2} M_{\mathrm{A}}\left(4 n M_{\mathrm{A}}+3 m M_{\mathrm{B}}\right) b^{2}\right. \\
& +m\left(4 n^{2} M_{\mathrm{A}}^{2}+6 m n M_{\mathrm{A}} M_{\mathrm{B}}\right. \\
& \left.\left.+m^{2} M_{\mathrm{B}}^{2}\right) b^{\prime 2}\right\} / 6\left(2 n M_{\mathrm{A}}+m M_{\mathrm{B}}\right)^{2}
\end{aligned}
$$

where $M_{\mathrm{A}}$ and $M_{\mathrm{B}}$ are the segment masses of polymers $\mathrm{A}$ and $\mathrm{B}$, respectively. For a homopolymer $\left(b^{\prime}=b, M_{\mathrm{A}}=M_{\mathrm{B}}\right)$, the value of eq 4 should be equal to the exact value of $(m+2 n) b^{2} / 6$, independent of the choice of $m$ and $n$. The ratio of the value of eq 4 to the exact one becomes

$$
1-2 a /(a+2)^{3}
$$

where $a=m / n$. This depends on $a$ and is not in general equal to unity. However, it is between 1 and $25 / 27$, the minimum value at $a=1$, and we can consider this model as rather suitable in our approximate treatment.

\section{THE PROBABILITY DENSITIES}

In order to discuss the statistical behavior of the model, we must consider the normalized probability density of the occurrence of the center of a side block at a position $r$ from that of the central block. Taking the center of mass of the central block as the origin of the coordinate system, the end of the vector $r$ moves in the shell between two spheres of radius $B-A$ and $B+A$ (Figure 2). Point $\mathrm{P}$, the end of vector $B$, moves with equal weight on a spherical surface of radius $B$ centered around point O. Point $\mathrm{Q}$, the end of vector $\boldsymbol{A}$, moves on a spherical surface of radius $A$ centered around point $\mathrm{P}$ with equal weight on the surface. Taking $\boldsymbol{B}$ to be fixed, a conformation is determined by one position of $\mathrm{Q}$. The shell between two spheres of radius $r$ and $r+\mathrm{d} r$ intersects the spherical surface $P$ resulting in the

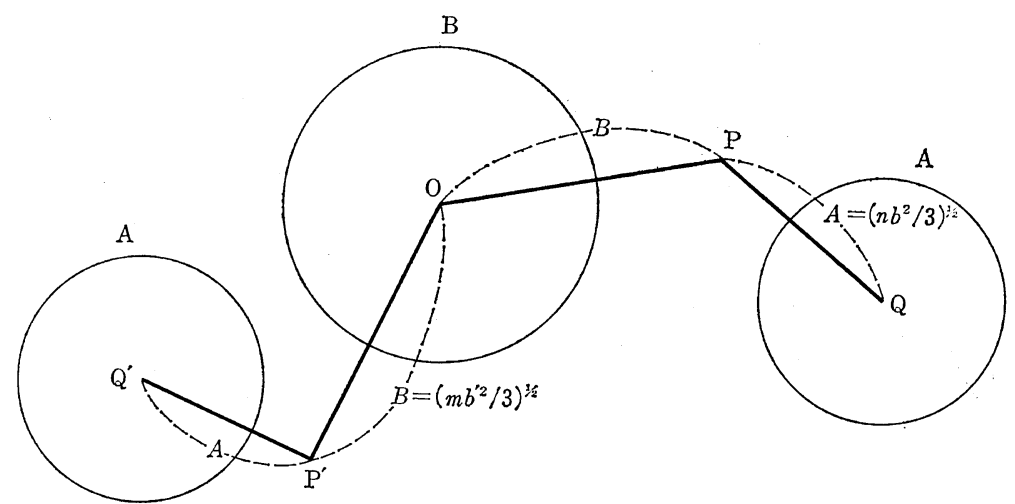

Figure 1. A triblock copolymer which is represented by three spherical segmental distributions connected to each other by four sticks. 


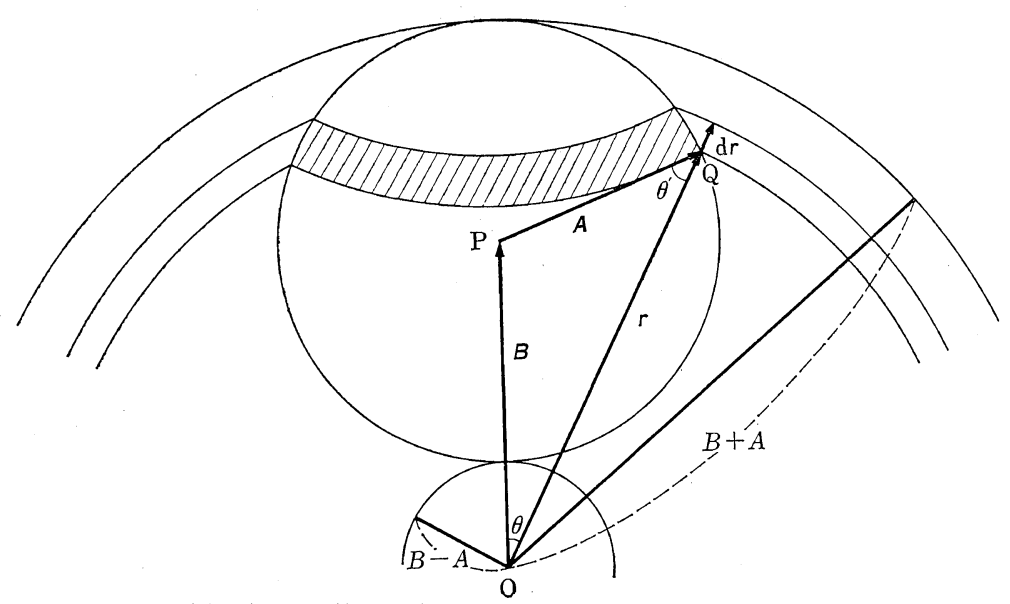

Figure 2. The vanishingly small hatched zonal area where the shell between two spheres of radius $r$ and $r+d r$ and the sphere $\mathrm{P}$ intersect.

zonal area $2 \pi r \cdot \mathrm{d} r \cdot A / B$ (see Appendix). Viewed from point $\mathrm{O}$, the probability that $\mathrm{Q}$ occurs at a position $r$ is in proportion to this vanishingly small hatched zonal area. The occurrence probability density per unit volume is proportional to $A / 2 B r$. So the normalized probability density of the occurrence of a side-block center at a position $r$ is given by

$$
1 /(8 \pi A B r)
$$

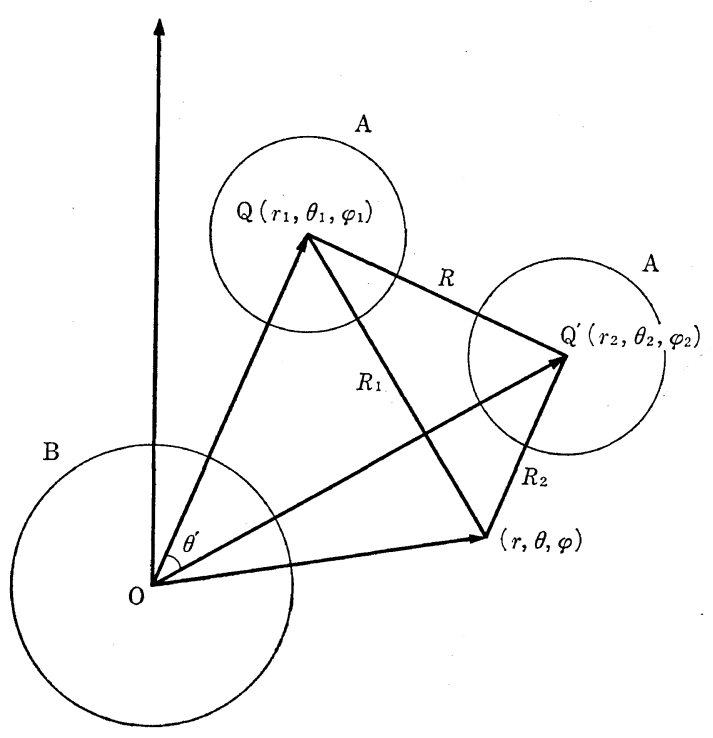

Figure 3. The positions of the two side blocks taking the center of mass of the central block as the origin of the coordinate system.
It can be shown that this result is applicable to both the case of $B>A$ and of $A>B$.

On the assumption of a Gaussian distribution of the segments around the center of mass of each chain block, the normalized probability density for polymer segments at a position $(r, \vartheta, \varphi)$ in a fixed conformation is given by

$$
\rho(r, \vartheta, \varphi)=\nu_{\mathrm{A}}+\nu_{\mathrm{B}}
$$

where

$$
\begin{aligned}
& \nu_{\mathrm{A}}=\frac{1}{2}\left(\frac{9}{\pi n b^{2}}\right)^{3 / 2}\left\{\exp \left[-\frac{9 R_{1}{ }^{2}}{n b^{2}}\right]+\exp \left[-\frac{9 R_{2}{ }^{2}}{n b^{2}}\right]\right\} \\
& \nu_{\mathrm{B}}=\left(\frac{9}{\pi m b^{\prime 2}}\right)^{3 / 2} \exp \left[-\frac{9 r^{2}}{n b^{2}}\right]
\end{aligned}
$$

are the normalized probability densities of the polymer segments A and B (see Figure 3).

\section{THE PARTITION FUNCTION AND THE FREE ENERGY}

The partition function $Z$ for a small volume element $\mathrm{d} V$ is evaluated by Bragg-Williams approximation with the lattice model. The sizes of an A-segment, a B-segment, and a solvent molecules $\mathrm{C}$ are all assumed to be the same and a lattice cell corresponds to either a segment or a solvent molecule.

The interaction energies between polymer segments and solvent molecules $\mathrm{AA}, \mathrm{BB}, \mathrm{CC}, \mathrm{AB}$, $\mathrm{BC}$, and $\mathrm{CA}$ are denoted by

$$
\varepsilon_{11}, \varepsilon_{22}, \varepsilon_{33}, \varepsilon_{12}, \varepsilon_{23} \text {, and } \varepsilon_{31} \text {. }
$$

If the volume element $\mathrm{d} V$ consists of $N_{1}$ A- 
segments, $N_{2}$ B-segments, and $N_{3}$ solvent molecules C $\left(N=N_{1}+N_{2}+N_{3}\right)$, and the numbers of the pairs are

$$
\begin{aligned}
& M_{11}, M_{22}, M_{33}, M_{12} \equiv(z-2) X_{3}, \\
& M_{23}=(z-2) X_{1}, M_{31} \equiv(z-2) X_{2}
\end{aligned}
$$

with the coordination number $z$, then the total potential energy $\Delta E$ in $\mathrm{d} V$ can be written as

$$
\begin{aligned}
\Delta E= & (z-2)\left\{\left(N_{1} \varepsilon_{11}+N_{2} \varepsilon_{22}+N_{3} \varepsilon_{33}\right) / 2\right. \\
& \left.+\left(X_{3} \Delta \varepsilon_{12}+X_{1} \Delta \varepsilon_{23}+X_{2} \Delta \varepsilon_{31}\right)\right\}
\end{aligned}
$$

where

$$
\Delta \varepsilon_{i j}=\Delta \varepsilon_{j i}=\varepsilon_{i j}-\left(\varepsilon_{i i}+\varepsilon_{j j}\right) / 2
$$

is the excess interaction energy for forming an $i-j$ pair.

By the Bragg-Williams approximation the partition function $Z$ in $\mathrm{d} V$ becomes

$$
\begin{aligned}
Z= & \left(\frac{z-1}{N}\right)^{N_{1}+N_{2}} \frac{N !}{N_{3} !} \\
& \times \exp \left[-\frac{z-2}{2 k T}\left(N_{1} \varepsilon_{11}+N_{2} \varepsilon_{22}+N_{3} \varepsilon_{33}\right)\right. \\
& \left.-\frac{z-2}{k T}\left(\frac{N_{1} N_{2}}{N} \Delta \varepsilon_{12}+\frac{N_{2} N_{3}}{N} \Delta \varepsilon_{23}+\frac{N_{3} N_{1}}{N} \Delta \varepsilon_{31}\right)\right]
\end{aligned}
$$

The free-energy density $f$ in this volume element is given by

$$
f \mathrm{~d} V=-k T \ln Z
$$

The mixing free-energy density $\Delta f_{\mathrm{M}}$, the remainder of $f$ after the summation of pure ones has been subtracted, is

$$
\begin{aligned}
\Delta f_{\mathrm{M}} \mathrm{d} V= & (z-2)\left(\frac{N_{1} N_{2}}{N} \Delta \varepsilon_{12}+\frac{N_{2} N_{3}}{N} \Delta \varepsilon_{23}\right. \\
& \left.+\frac{N_{3} N_{1}}{N} \Delta \varepsilon_{31}\right)+k T \ln \frac{N_{3}}{N} \\
= & \left\{2 n\left(\theta_{1}-k T\right) \nu_{\mathrm{A}}+m\left(\theta_{2}-k T\right) \nu_{\mathrm{B}}\right. \\
& +4 n^{2} l^{3}\left(-\theta_{1}+k T / 2\right) \nu_{\mathrm{A}}^{2} \\
& +m^{2} l^{3}\left(-\theta_{2}+k T / 2\right) \nu_{\mathrm{B}}^{2} \\
& \left.+2 m n l^{3}\left(\theta_{12}-\theta_{1}-\theta_{2}+k T\right) \nu_{\mathrm{A}} \nu_{\mathrm{B}}\right\} \\
& \times r^{2} \sin \vartheta \mathrm{d} r \mathrm{~d} \vartheta \mathrm{d} \varphi
\end{aligned}
$$

where we use the relations

$$
\begin{gathered}
N_{1}=2 n \nu_{\mathrm{A}} \mathrm{d} V, \quad N_{2}=m \nu_{\mathrm{B}} \mathrm{d} V, \quad N=\mathrm{d} V / l^{3}, \\
\mathrm{~d} V=r^{2} \sin \vartheta \mathrm{d} r \mathrm{~d} \vartheta \mathrm{d} \varphi
\end{gathered}
$$

with the lattice constant $l$. We denote the $\theta$ value for each pair by

$\theta_{1} \equiv(z-2) \Delta \varepsilon_{31}, \quad \theta_{2} \equiv(z-2) \Delta \varepsilon_{23}, \quad \theta_{12} \equiv(z-2) \Delta \varepsilon_{12}$
Substituting eq 7 into $\nu_{\mathrm{A}}$ and $\nu_{\mathrm{B}}$ and integrating $\Delta f_{M}$ over the whole space, the total mixing free-energy $\Delta F_{\mathrm{M}}$ at a fixed conformation becomes

$$
\begin{aligned}
\Delta F_{\mathrm{M}}= & \int_{0}^{\infty} \int_{0}^{\pi} \int_{0}^{2 \pi} \Delta f_{\mathrm{M}} \mathrm{d} V \\
= & 2 n\left(\theta_{1}-k T\right)+m\left(\theta_{2}-k T\right) \\
& +\frac{1}{\sqrt{2}} n^{2} l^{3}\left(-\theta_{1}+k T / 2\right)\left(9 / \pi n b^{2}\right)^{3 / 2} \\
& \times\left\{1+\exp \left[-9 R^{2} / 2 n b^{2}\right]\right\} \\
& +\frac{1}{2 \sqrt{2}} m^{2} l^{3}\left(-\theta_{2}+k T / 2\right)\left(9 / \pi m b^{2}\right)^{3 / 2} \\
& +27 m n l^{3}\left(\theta_{12}-\theta_{1}-\theta_{2}+k T\right) \\
& \times \pi^{-3 / 2}\left(n b^{2}+m b^{\prime 2}\right)^{-3 / 2} \\
& \times \exp \left[-9 r_{1}^{2} /\left(n b^{2}+m b^{\prime 2}\right)\right] \\
& \left.+\exp \left[-9 r_{2}^{2} /\left(n b^{2}+m b^{\prime 2}\right)\right]\right\}
\end{aligned}
$$

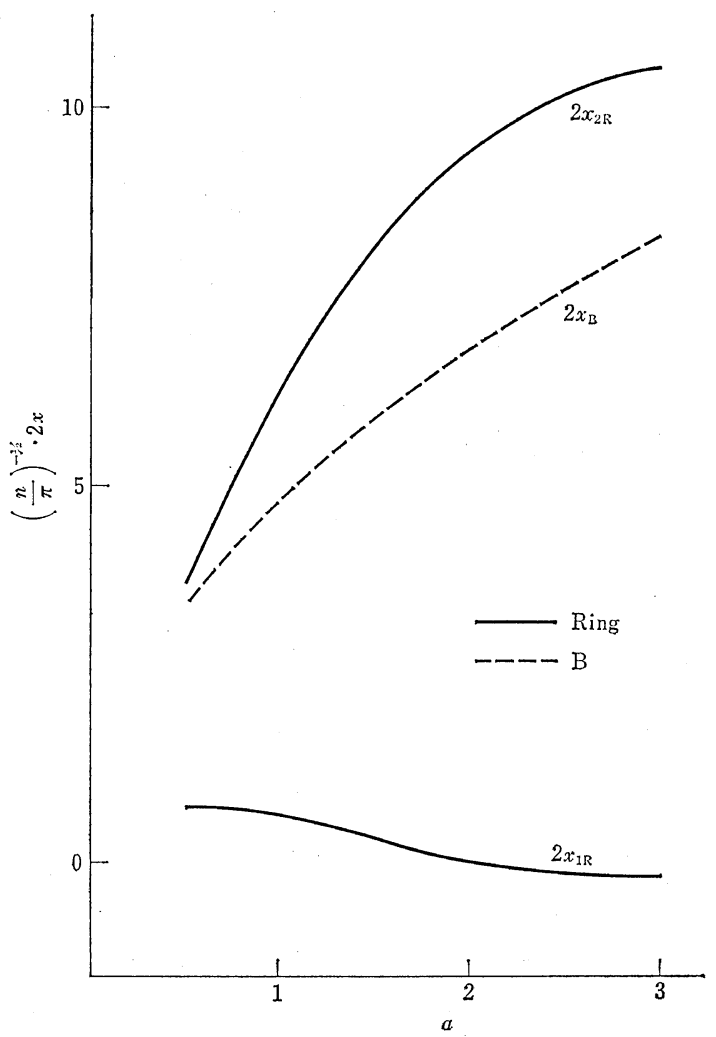

Figure 4. The volume expansion factors expanded around unity for $m=a n$ at $T=303^{\circ} \mathrm{K}$ with $\Theta_{1}=308^{\circ} \mathrm{K}$, $\Theta_{2}=0^{\circ} \mathrm{K}$, and $\Theta_{12}=303^{\circ} \mathrm{K} \sim 350^{\circ} \mathrm{K}$. The subscription $\mathrm{R}$ indicates a ring conformation. 
where

$$
R^{2}=r_{1}^{2}+r_{2}^{2}-2 r_{1} r_{2} \cos \vartheta^{\prime}
$$

Making use of eq 6 for the normalized probability densities of the position of each center of the blocks, after introduction of the volumeexpansion factors of the blocks $A$ and block $B$ in the solution, $\alpha_{1}$ and $\alpha_{2}\left(b \rightarrow \alpha_{1} b, b^{\prime} \rightarrow \alpha_{2} b^{\prime}\right)$, the conformationally averaged mixing free-energy $\Delta F_{\mathrm{MC}}$ is obtained as

$$
\begin{aligned}
\Delta F_{\mathrm{MC}}= & \iint_{|A-B|}^{|A+B|} \iint_{0}^{\pi} \iint_{0}^{2 \pi}\left(\frac{1}{8 \pi A B}\right)^{2} \frac{1}{r_{1} r_{2}} \Delta F_{\mathrm{M}} r_{1}^{2} \sin \vartheta_{1} \mathrm{~d} r_{1} \mathrm{~d} \vartheta_{1} \mathrm{~d} \varphi_{1} r_{2}^{2} \sin \vartheta_{2} \mathrm{~d} r_{2} \mathrm{~d} \vartheta_{2} \mathrm{~d} \varphi_{2} \\
= & 2 n\left(\theta_{1}-k T\right)+m\left(\theta_{2}-k T\right)+9 n^{2} l^{3}\left(-\theta_{1}+k T / 2\right) / \sqrt{6} \pi^{3 / 2} A^{3} \alpha_{1}^{3} \\
& +\left(n^{2} l^{3} / 8 \pi^{3 / 2}\right)\left(-\theta_{1}+k T / 2\right) / A^{2} B^{2} \alpha_{1}^{2} \alpha_{2}^{2}\left[( A \alpha _ { 1 } / \sqrt { 6 } ) \left\{2 \mathrm{e}^{-6}-2+2 \mathrm{e}^{-6\left(B^{2} \alpha_{2}{ }^{2} / A^{2} \alpha_{1}{ }^{2}\right)}-\mathrm{e}^{-6\left(B \alpha_{2} / A \alpha_{1}-1\right) 2}\right.\right. \\
& \left.-\mathrm{e}^{\left.-6 \mid B \alpha_{2} / A \alpha_{1}+1\right) 2}\right\}+2\left\{2 A \alpha_{1} \int_{0}^{\sqrt{6}} \mathrm{e}^{-t^{2}} \mathrm{~d} t+2 B \alpha_{2} \int_{0}^{\sqrt{6}\left(B \alpha_{2} / A \alpha_{1}\right)} \mathrm{e}^{-t^{2}} \mathrm{~d} t+\left(A \alpha_{1}-B \alpha_{2}\right) \int_{0}^{\sqrt{6}\left(B \alpha_{2} / A \alpha_{1}-1\right)} \mathrm{e}^{-t 2} \mathrm{~d} t\right. \\
& \left.\left.-\left(A \alpha_{1}+B \alpha_{2}\right) \int_{0}^{\sqrt{6}\left(B \alpha_{2} / A \alpha_{1}+1\right)} \mathrm{e}^{-t^{2}} \mathrm{~d} t\right\}\right]+\left(9 m^{2} l^{3} / 2 \sqrt{6} \pi^{3 / 2}\right)\left(-\theta_{2}+k T / 2\right) / B^{3} \alpha_{2}{ }^{3} \\
& +\left(\sqrt{3} m n l^{3} / 2 \pi^{3 / 2}\right)\left(\theta_{12}-\theta_{1}-\theta_{2}+k T\right) / A B \alpha_{1} \alpha_{2}\left(A^{2} \alpha_{1}{ }^{2}+B^{2} \alpha_{2}{ }^{2}\right)^{1 / 2} \\
& \times\left\{\exp \left[-3\left(A \alpha_{1}-B \alpha_{2}\right)^{2} /\left(A^{2} \alpha_{1}{ }^{2}+B^{2} \alpha_{2}{ }^{2}\right)\right]-\exp \left[-3\left(A \alpha_{1}+B \alpha_{2}\right)^{2} /\left(A^{2} \alpha_{1}{ }^{2}+B^{2} \alpha_{2}{ }^{2}\right)\right]\right\}
\end{aligned}
$$

Apart from this mixing free-energy, there is another component of the free energy, that is, the excess elastic free-energy $\Delta F_{\mathrm{el}}$ due to the conformational entropy. According to Flory's theory we have

$$
\begin{aligned}
\Delta F_{\mathrm{e} 1}= & 2 \times \frac{3}{2} k T\left(\alpha_{1}{ }^{2}-1\right)-2 k T \ln {\alpha_{1}}^{3} \\
& +\frac{3}{2} k T\left(\alpha_{2}{ }^{2}-1\right)-k T \ln {\alpha_{2}}^{3}
\end{aligned}
$$

The total free-energy change $\Delta F$ due to dissolution is given by

$$
\Delta F=\Delta F_{\mathrm{MC}}+\Delta F_{\mathrm{el}}
$$

\section{THE VOLUME-EXPANSION FACTOR}

The values of $\alpha_{1}$ and $\alpha_{2}$ can be determined where by the minimum condition on the free energy

$$
\partial \Delta F / \partial \alpha_{1}=\partial \Delta F / \partial \alpha_{2}=0
$$

Unfortunately $\Delta F_{\mathrm{MC}}$ is so complicated that eq 18 cannot be solved exactly without any approximation. Therefore we are compelled to use the linear approximation with respect to $\left(\alpha_{1}-1\right)$ and $\left(\alpha_{2}-1\right)$. After the calculation the solution may be rounded into the well-known Flory's expression. Carrying out a lengthy and tedious calculation, we have

$$
\begin{aligned}
& \alpha_{1}{ }^{5}-\alpha_{1}{ }^{3}=\psi_{1}\left(1-\Theta_{1}{ }^{*} / T\right) \\
& \alpha_{2}{ }^{5}-\alpha_{2}{ }^{3}=\psi_{2}\left(1-\Theta_{2}{ }^{*} / T\right)
\end{aligned}
$$

$$
\begin{aligned}
& \psi_{1}=\left(P_{1}+Q_{1}\right) / 12, \quad \phi_{2}=\left(P_{2}+Q_{2}+2 R_{2}\right) / 6 \\
& \Theta_{1}^{*}=\left(P_{1} \Theta_{1}-Q_{1} \Theta_{12}\right) /\left(P_{1}+2 Q_{1}\right) \\
& \Theta_{2}{ }^{*}=\left(P_{2} \Theta_{1}+Q_{2} \Theta_{2}-R_{2} \Theta_{12}\right) /\left(P_{2}+Q_{2}+2 R_{2}\right) \\
& \Theta_{1}=2 \theta_{1} / k, \quad \Theta_{2}=2 \theta_{2} / k, \quad \Theta_{12}=2\left(\theta_{12}-\theta_{1}-\theta_{2}\right) / k \\
& P_{1}=9 \sqrt{6} n^{2} l^{3} / 2 \pi^{3 / 2} A^{3}+\left(n^{2} l^{3} / 8 \pi^{3 / 2} A^{2} B^{2}\right)\left[(A / \sqrt{6})\left\{2 \mathrm{e}^{-6}-2+2 \mathrm{e}^{-6\left(B^{2} / A^{2}\right)}-\mathrm{e}^{-6(B / A-1) 2}-\mathrm{e}^{-6(B / A+1) 2}\right\}\right. \\
& \left.+2\left\{2 A \int_{0}^{\sqrt{6}} \mathrm{e}^{-t^{2}} \mathrm{~d} t+4 B \int_{0}^{\sqrt{6}(B / A)} \mathrm{e}^{-t^{2}} \mathrm{~d} t+(A-2 B) \int_{0}^{\sqrt{6}(B / A-1)} \mathrm{e}^{-t^{2}} \mathrm{~d} t-(A+2 B) \int_{0}^{\sqrt{6}(B / A+1)} \mathrm{e}^{-t^{2}} \mathrm{~d} t\right\}\right] \\
& Q_{1}=\left\{\sqrt{3} m n l^{3} / 2 \pi^{3 / 2} A B\left(A^{2}+B^{2}\right)^{5 / 2}\right\}\left(\left\{\left(2 A^{2}+B^{2}\right)\left(A^{2}+B^{2}\right)+6 A B\left(A^{2}-B^{2}\right)\right\} \exp \left[-3(A-B)^{2} /\left(A^{2}+B^{2}\right)\right]\right. \\
& \left.+\left\{-\left(2 A^{2}+B^{2}\right)\left(A^{2}+B^{2}\right)+6 A B\left(A^{2}-B^{2}\right)\right\} \exp \left[-3(A+B)^{2} /\left(A^{2}+B^{2}\right)\right]\right)
\end{aligned}
$$




$$
\begin{aligned}
P_{2}= & \left(n^{2} l^{3} / 4 \pi^{3 / 2} A^{2} B^{2}\right)\left[(A / \sqrt{6})\left\{2 \mathrm{e}^{-6}-2+2 \mathrm{e}^{-6\left(B^{2} / A^{2}\right)}-\mathrm{e}^{-6(B / A-1) 2}-\mathrm{e}^{-6(B / A+1) 2}\right\}\right. \\
& \left.+4 A \int_{0}^{\sqrt{6}} \mathrm{e}^{-t^{2}} \mathrm{~d} t+2 B \int_{0}^{\sqrt{6}(B / A)} \mathrm{e}^{-t^{2}} \mathrm{~d} t+(2 A-B) \int_{0}^{\sqrt{6}(B / A-1)} \mathrm{e}^{-t^{2}} \mathrm{~d} t-(2 A+B) \int_{0}^{\sqrt{6}(B / A+1)} \mathrm{e}^{-t^{2}} \mathrm{~d} t\right] \\
Q_{2}= & 9 \sqrt{6} m^{2} l^{3} / 4 \pi^{3 / 2} B^{3} \\
R_{2}= & -\left\{\sqrt{3} m n l^{3} / 2 \pi^{3 / 2} A B\left(A^{2}+B^{2}\right)^{5 / 2}\right\}\left(\left\{-\left(A^{2}+2 B^{2}\right)\left(A^{2}+B^{2}\right)+6 A B\left(A^{2}-B^{2}\right)\right\}\right. \\
& \left.\times \exp \left[-3(A-B)^{2} /\left(A^{2}+B^{2}\right)\right]+\left\{\left(A^{2}+2 B^{2}\right)\left(A^{2}+B^{2}\right)+6 A B\left(A^{2}-B^{2}\right)\right\} \exp \left[-3(A+B)^{2} /\left(A^{2}+B^{2}\right)\right]\right)
\end{aligned}
$$

In terms of eq 4 and 19, the volume-expansion factor for the whole block copolymer is calculated to be

$$
\alpha^{2}=\frac{2 n^{2} M_{\mathrm{A}}\left(4 n M_{\mathrm{A}}+3 m M_{\mathrm{B}}\right) b^{2} \alpha_{1}^{2}+m\left(4 n^{2} M_{\mathrm{A}}^{2}+6 m n M_{\mathrm{A}} M_{\mathrm{B}}+m^{2} M_{\mathrm{B}}^{2}\right) b^{\prime 2} \alpha_{2}^{2}}{2 n^{2} M_{\mathrm{A}}\left(4 n M_{\mathrm{A}}+3 m M_{\mathrm{B}}\right) b^{2}+m\left(4 n^{2} M_{\mathrm{A}}^{2}+6 m n M_{\mathrm{A}} M_{\mathrm{B}}+m^{2} M_{\mathrm{B}}^{2}\right) b^{\prime 2}}
$$

For the special case of a homopolymer $\mathrm{B}$, eq 19 results in

$$
\alpha_{\mathrm{B}}^{5}-\alpha_{\mathrm{B}}{ }^{3}=27 \times 2^{-5 / 2} \pi^{-3 / 2} m^{1 / 2}\left(1-\Theta_{2} / T\right)
$$

This is, of course, just the same as Flory's result.

\section{DISCUSSION}

Below the $\theta$ temperature for the side blocks, they may coagulate to make the whole copolymer a ring and so restrict the whole conformation into a smaller radius of gyration. In order to discuss this point, the excess free-energy $\Delta F_{\mathrm{R}}$ in the ring conformation is evaluated and compared with the average free-energy $\Delta F$ for all conformations.

The free energy in the ring conformation is given by

$$
\begin{aligned}
\Delta F_{\mathrm{R}}= & 2 n\left(\theta_{1}-k T\right)+m\left(\theta_{2}-k T\right) \\
& +\left(3 \sqrt{6} n^{2} l^{3} / \pi^{3 / 2}\right)\left(-\theta_{1}+k T / 2\right) / A^{3} \alpha_{1 \mathrm{R}}^{3} \\
& +\left(3 \sqrt{6} m^{2} l^{3} / 4 \pi^{3 / 2}\right)\left(-\theta_{2}+k T / 2\right) / B^{3} \alpha_{2 \mathrm{R}}^{3} \\
& +\left(\sqrt{3} m n l^{3} / 2 \pi^{3 / 2}\right)\left(\theta_{12}-\theta_{1}-\theta_{2}+k T\right) \\
& \mid A B \alpha_{1 \mathrm{R}} \alpha_{2 \mathrm{R}}\left(A^{2} \alpha_{1 \mathrm{R}}^{2}+B^{2} \alpha_{2 \mathrm{R}}^{2}\right)^{1 / 2} \\
& \times\left\{\exp \left[-3\left(A \alpha_{1 \mathrm{R}}-B \alpha_{2 \mathrm{R}}\right)^{2} /\left(A^{2} \alpha_{1 \mathrm{R}}^{2}+B^{2} \alpha_{2 \mathrm{R}}^{2}\right)\right]\right. \\
& \left.-\exp \left[-3\left(A \alpha_{1 \mathrm{R}}+B \alpha_{2 \mathrm{R}}\right)^{2} /\left(A^{2} \alpha_{1 \mathrm{R}}^{2}+B^{2} \alpha_{2 \mathrm{R}}^{2}\right)\right]\right\} \\
& +2 \times \frac{3}{2} k T\left(\alpha_{1 \mathrm{R}}^{2}-1\right)-2 k T \ln \alpha_{1 \mathrm{R}}^{3} \\
& +\frac{3}{2} k T\left(\alpha_{2 \mathrm{R}}^{2}-1\right)-k T \ln \alpha_{2 \mathrm{R}}^{3}
\end{aligned}
$$

where $\alpha_{1 \mathrm{R}}$ and $\alpha_{2 \mathrm{R}}$ are volume-expansion factors of the blocks $\mathrm{A}$ and the block $\mathrm{B}$ in the ring conformation. Equation 23 gives a special case where $r_{1}=r_{2}, \vartheta_{1}=\vartheta_{2}$, and $\varphi_{1}=\varphi_{2}$, as shown in Figure 3.

$\Delta F_{\mathrm{R}}$ is different from $\Delta F$ only in the term proportional to the square of the segmental density $\nu_{A}$ of the block A.

It is not so difficult to see that $\Delta F_{\mathrm{R}}$ is certainly smaller than $\Delta F$ below the $\theta$ temperature for the side blocks, because the volumeexpansion factors near the $\theta$ state can be regarded as close to unity and the exponential terms and the error functions in the coefficient of $\left(-\theta_{1}+k T / 2\right)$ in $\Delta F_{\mathrm{MC}}$ are much smaller than the others. An ABA-type triblock copolymer, in such a case, would reasonably make a ring with the following volume-expansion factors $\alpha_{1 \mathrm{R}}$ and $\alpha_{2 \mathrm{R}}$

$$
\begin{aligned}
& \alpha_{1 \mathrm{R}}^{5}-\alpha_{1 \mathrm{R}}^{3}=\phi_{1 \mathrm{R}}\left(1-\Theta_{1 \mathrm{R}}^{*} / T\right) \\
& \alpha_{2 \mathrm{R}}^{5}-\alpha_{2 \mathrm{R}}^{3}=\psi_{2 \mathrm{R}}\left(1-\Theta_{2 \mathrm{R}}^{*} / T\right)
\end{aligned}
$$

where

$$
\begin{aligned}
& \psi_{1 \mathrm{R}}=\left(P_{1 \mathrm{R}}+2 Q_{1 \mathrm{R}}\right) / 12, \quad \phi_{2 \mathrm{R}}=\left(Q_{2 \mathrm{R}}+2 R_{2 \mathrm{R}}\right) / 6 \\
& \Theta_{1 \mathrm{R}}^{*}=\left(P_{1 \mathrm{R}} \Theta_{1}-Q_{1 \mathrm{R}} \Theta_{12}\right) /\left(P_{1 \mathrm{R}}+2 Q_{1 \mathrm{R}}\right) \\
& \Theta_{2 \mathrm{R}}^{*}=\left(Q_{2 \mathrm{R}} \Theta_{2}-R_{2 \mathrm{R}} \Theta_{12}\right) /\left(Q_{2 \mathrm{R}}+2 R_{2 \mathrm{R}}\right) \\
& P_{1 \mathrm{R}}=9 \sqrt{6} n^{2} l^{3} / \pi^{3 / 2} A^{3}, \quad Q_{1 \mathrm{R}}=Q_{1} \\
& P_{2 \mathrm{R}}=0, \quad Q_{2 \mathrm{R}}=Q_{2}, \quad R_{2 \mathrm{R}}=R_{2}
\end{aligned}
$$

The unperturbed radius of gyration in the ring conformation for this model is calculated to be

$$
\left\langle s^{2}\right\rangle_{\mathrm{R}}=\frac{4 n^{2} M_{\mathrm{A}}\left(6 n M_{\mathrm{A}}+5 m M_{\mathrm{B}}\right) b^{2}+m^{2} M_{\mathrm{B}}\left(14 n M_{\mathrm{A}}+3 m M_{\mathrm{B}}\right) b^{\prime 2}+4 n m M_{\mathrm{A}} M_{\mathrm{B}}\left|m b^{\prime 2}-n b^{2}\right|}{18\left(2 n M_{\mathrm{A}}+m M_{\mathrm{B}}\right)^{2}}
$$




\section{H. ENDo and M. Yamamoto}

The volume-expansion factor for the whole block copolymer in the ring conformation becomes $\alpha_{\mathrm{R}}{ }^{2}=\frac{4 n^{2} M_{\mathrm{A}}\left(6 n M_{\mathrm{A}}+5 m M_{\mathrm{B}}\right) b^{2} \alpha_{1 \mathrm{R}}^{2}+m^{2} M_{\mathrm{B}}\left(14 n M_{\mathrm{A}}+3 m M_{\mathrm{B}}\right) b^{\prime 2} \alpha_{2 \mathrm{R}}^{2}+4 n m M_{\mathrm{A}} M_{\mathrm{B}}\left|m b^{\prime 2} \alpha_{2 \mathrm{R}}^{2}-n b^{2} \alpha_{1 \mathrm{R}}^{2}\right|}{4 n^{2} M_{\mathrm{A}}\left(6 n M_{\mathrm{A}}+5 m M_{\mathrm{B}}\right) b^{2}+m^{2} M_{\mathrm{B}}\left(14 n M_{\mathrm{A}}+3 m M_{\mathrm{B}}\right) b^{\prime 2}+4 n m M_{\mathrm{A}} M_{\mathrm{B}}\left|m b^{\prime 2}-n b^{2}\right|}$

From eq 4 and 26 , the ratio of $\left\langle s^{2}\right\rangle_{R}$ to $\left\langle s^{2}\right\rangle$ for this model is given by

$$
\frac{\left\langle s^{2}\right\rangle_{\mathrm{R}}}{\left\langle s^{2}\right\rangle}=\frac{3 a^{3}+14 a^{2}+20 a+24+4 a|a-1|}{3\left(a^{3}+6 a^{2}+10 a+8\right)}
$$

where $b=b^{\prime}, M_{\mathrm{A}}=M_{\mathrm{B}}$, and $m=a n$. This is between 1 and the minimum value $61 / 75$ at $a=1$.

Experiments were made with nearly equimolar block copolymers of the ABA-type (poly(methyl methacrylate) - polystyrene - poly(methyl methacrylate)) at $30.0^{\circ} \mathrm{C}$ in $p$-xylene whose $\theta$ temperature for the side blocks $\mathrm{A}$ is $35^{\circ} \mathrm{C} .{ }^{9}$

Expanding the volume-expansion factors around unity, eq 19 and 24 are reduced to

$$
\begin{aligned}
& 2 x_{1}=\Psi_{1}\left(1-\Theta_{1}{ }^{*} / T\right), \quad 2 x_{2}=\Psi_{2}\left(1-\Theta_{2}{ }^{*} / T\right) \\
& 2 x_{1 \mathrm{R}}=\Psi_{1 \mathrm{R}}\left(1-\Theta_{1 \mathrm{R}}^{*} / T\right), \quad 2 x_{2 \mathrm{R}}=\Psi_{2 \mathrm{R}}\left(1-\Theta_{2 \mathrm{R}}^{*} / T\right)
\end{aligned}
$$

The results of substitution of the values of eq 25 for $m=a n$ into eq 29 at $\Theta_{1}=308^{\circ} \mathrm{K}$ and $T=303^{\circ} \mathrm{K}$ are shown in Figure 4 for the various values of $a$. As $p$-xylene is a moderate solvent for the central polymer, polystyrene, $\Theta_{2}$ is assumed to be $0^{\circ} \mathrm{K}$. It is reasonable to regard $2 \theta_{12} / k$, the polymer-polymer $\theta$ temperature, as fairly high, but it makes little difference in each volume-expansion factor whether $2 \theta_{12} / k$ is taken to be $303^{\circ} \mathrm{K}$ or $350^{\circ} \mathrm{K}$.

Below $a=1, \alpha_{2 \mathrm{R}}$ is slightly smaller than $\alpha_{2}$, while the volume-expansion factor in the ring conformation of a isolated chain is generally larger than that of the linear one. ${ }^{13}$ This is because of the existence of the side-block copolymers which are immersed in their $\theta$ solvent. Unlike the general isolated chain also, $2 X_{1}$ and $2 X_{1 \mathrm{R}}$ are not always negative below their $\theta$ temperature, because of the intervention of the other parameters, $\Theta_{2}$ and $\Theta_{12}$.

The intrinsic viscosities $[\eta]_{\mathrm{B}}$ of the parent homopolymer $\mathbf{B}$ and $[\eta]_{\mathrm{R}}$ for the ring conformation are evaluated by eq $2,22,26$, and 27 , assuming that $b \simeq b^{\prime}$ and $M_{\mathrm{A}} \simeq M_{\mathrm{B}}$, due to the nearly equal molecular weight $M_{0}$ of each monomer of methyl methacrylate and styrene.

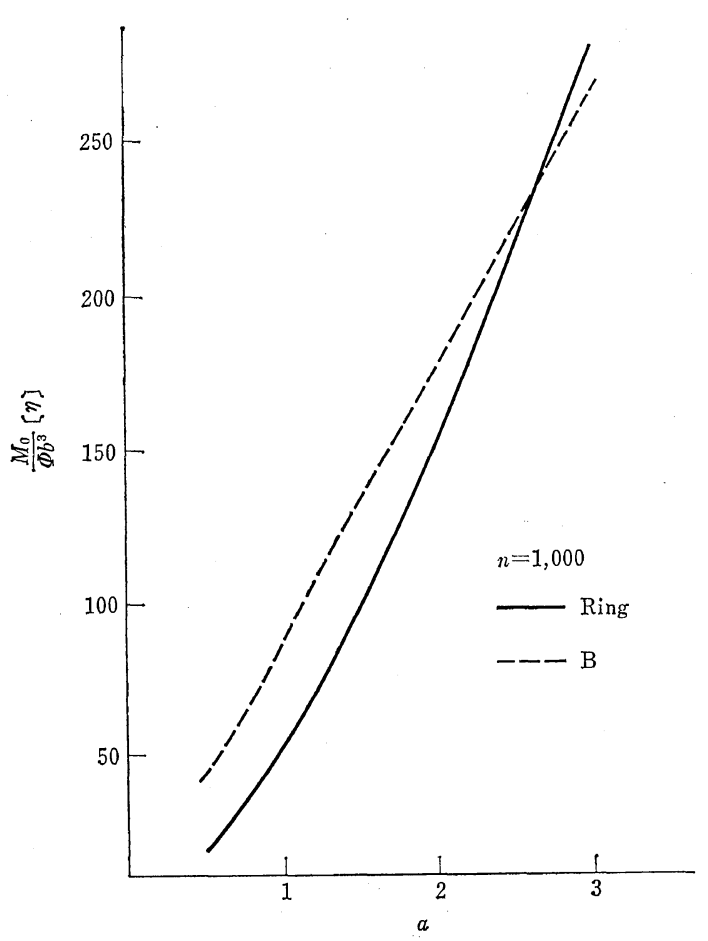

Figure 5. The intrinsic viscosities $\frac{M_{0}}{\Phi b^{3}}[\eta]_{\mathrm{B}}$ of the parent homopolymer $\mathrm{B}$ and $\frac{M_{0}}{\Phi b^{2}}[\eta]_{\mathrm{R}}$ for the ring conformation at $T=303^{\circ} \mathrm{K}$ with $\Theta_{1}=308^{\circ} \mathrm{K}, \Theta_{2}=$ $0^{\circ} \mathrm{K}$, and $\Theta_{12}=303^{\circ} \mathrm{K} \sim 350^{\circ} \mathrm{K}$.

The numerical values of $[\eta]_{\mathrm{B}}$ and $[\eta]_{\mathrm{R}}$ for the various values of $a$ are plotted in Figure 5 for $n=1000$, where $m=a n$ means that the molecular weight of the parent homopolymer $\mathrm{B}$ is $a \times n$ and that of the ABA block copolymer is $(a+2) n$.

Below $a \simeq 2.6,[\eta]_{\mathrm{R}}$ is always smaller than $[\eta]_{\mathrm{B}}$, which possesses a smaller molecular weight than $[\eta]_{R}$. This tendency is more emphasized with decreasing $a$ value and becomes most pronounced at $a=1$ where the ratio of $\left\langle s^{2}\right\rangle_{\mathrm{R}}$ to $\left\langle s^{2}\right\rangle$ comes to a minimum. Small $a$ values mean a large molecular-weight ratio of the side blocks $A$ to the central one and then the intramolecular attraction of the side polymers below the $\theta$ temperature becomes dominant and accelerates the decreasing of the intrinsic 
viscosity. The reason why even $[\eta]_{R}$ can not be smaller than $[\eta]_{\mathrm{B}}$ above $a \simeq 2.6$ seems to be that the side polymers' interaction can only make a slight contribution to the decrease of $[\eta]_{R}$ due to the small molecular-weight ratio of the side, though the conformational effect makes $[\eta]_{R}$ somewhat smaller.

It is reasonable to say, in this sense, that the intrinsic viscosity becomes smaller because of the small unperturbed radius of gyration in the ring conformation, apart from the effect of the interaction energy which would heighten the decrease of the viscosity, and that the side blocks $\mathrm{A}$ in the ring conformation restrict the allowable conformation of the central block B so much as to make the intrinsic viscosity of the whole block copolymer smaller than that of the precursor B below the vicinity of $a=2.6$. A similar tendency appears for the other values of $n$, e.g., 100 or 10000 .

\section{APPENDIX}

Increasing $r$ by an vanishingly small amount $\mathrm{d} r$, a shell between two spheres of radius $r$ and $r+\mathrm{d} r$ can be drawn. This shell and the sphere $P$ intersect at an infinitely small zonal area on the spherical surface of sphere $P$ (Figure 2). The width of this zone is $\mathrm{d} r / \sin \vartheta^{\prime}$. So this area becomes

$$
\int_{0}^{2 \pi} r \sin \vartheta \mathrm{d} \varphi \frac{\mathrm{d} r}{\sin \vartheta}=\frac{\sin \vartheta}{\sin \vartheta} 2 \pi r \mathrm{~d} r=\frac{A}{B} 2 \pi r \mathrm{~d} r
$$

The integral

$$
\int_{|B-A|}^{|B+A|} \frac{A}{B} 2 \pi r \mathrm{~d} r=4 \pi A^{2}
$$

gives the spherical surface area of sphere $P$.
This situation occurs at any point $Q$ on the spherical surface of radius $r$ centered around point $O$. The volume increase in the space due to $\mathrm{d} r$ is $4 \pi r^{2} \mathrm{~d} r$. Normalized by the spherical surface area $4 \pi A^{2}$ of sphere $\mathrm{P}$, the probability density per unit volume becomes

$$
\frac{A}{B} 2 \pi r \mathrm{~d} r \frac{1}{4 \pi A^{2}} \frac{1}{4 \pi r^{2} \mathrm{~d} r}=\frac{1}{8 \pi A B r}
$$

Acknowledgments One of the authors (H. E.) wishes to thank the Iwanami Fūjukai for its financial support.

\section{REFERENCES}

1. G. M. Burnett, D. Meares, and C. Paton, Trans. Faraday Soc., 58, 737 (1962).

2. S. Krause, J. Phys. Chem., 68, 1948 (1964).

3. J. R. Urwin and J.M. Stearne, Makromol. Chem., 78, 197, 204 (1964).

4. H. Inagaki, ibid., 86, 289 (1965).

5. H. Inagaki and T. Miyamoto, ibid., 87, 166 (1965).

6. D. Froelich and H. Benoit, ibid., 92, 224 (1966).

7. T. Inoue, T. Soen, H. Kawai, M. Fukatsu, and M. Kurata, J. Polym. Sci., Part B, 6, 75 (1968).

8. T. Kotaka, T. Tanaka, H. Ohnuma, Y. Murakami, and H. Inagaki, Polymer J., 1, 245 (1970).

9. H. Onuma, T. Kotaka, and H. Inagaki, ibid., 1, 716 (1970).

10. T. Tanaka and T. Kotaka, unpublished experiments.

11. P. J. Flory, "Principles of Polymer Chemistry," Cornell University Press, Ithaca, N. Y., 1953.

12. R. Chūjō and M. Yamamoto, unpublished work at Kyoto University.

13. E. F. Casassa, J. Polym. Sci., Part A, 3, 605 (1965). 